\title{
Soziale Probleme. Wissenssoziologische Überlegungen
}

\author{
Reiner Keller • Angelika Poferl
}

Eingegangen: 29. Oktober 2020 / Angenommen: 18. November 2020 / Online publiziert: 26. November 2020

(C) Der/die Autor(en) 2020

Zusammenfassung Der Beitrag widmet sich dem Verhältnis von Wissenssoziologie und Soziologie sozialer Probleme. Er geht von weitreichenden Überlappungen zwischen zentralen wissenssoziologischen Positionen und Forschungsinteressen sowie den im engeren und weiteren Sinne ,konstruktivistischen' Positionen innerhalb der Soziologie sozialer Probleme aus. Die Argumentation greift Konzepte aus der jüngeren deutschsprachigen Wissenssoziologie - „Problematisierungswissen“ und „Relevanzhorizonte“, „Wissenskulturen“ und „Wissensverhältnisse/Wissenspolitiken“ auf und verdeutlicht deren mögliche Anregungspotentiale für eine Soziologie sozialer Probleme.

\section{Social Problems. Reflections from a Sociology of Knowledge}

Abstract This chapter is devoted to the relationship between the sociology of knowledge and the sociology of social problems. It starts out from the assumption that there is a far-reaching overlap between central positions and research interests in the sociology of knowledge and 'constructionist' positions in the narrower and broader sense within the sociology of social problems. The argumentation takes up concepts from the more recent German-language sociology of knowledge- "knowledge of problematisation" and "relevance horizons", "knowledge cultures" and "knowledge relations/knowledge policies"-and illustrates their potentials for a sociology of social problems.

\section{R. Keller $(\bowtie)$}

Philosophisch-Sozialwissenschaftliche Fakultät, Universität Augsburg, Augsburg, Deutschland E-Mail: reiner.keller@phil.uni-augsburg.de

\section{A. Poferl}

Sozialwissenschaftliche Fakultät, Technische Universität Dortmund, Dortmund, Deutschland

E-Mail: angelika.poferl@tu-dortmund.de 


\section{Einleitung}

Im vorliegenden Beitrag möchten wir Überlegungen zum Verhältnis von Wissenssoziologie und Soziologie sozialer Probleme anstellen und einige konzeptuelle Vorschläge der jüngeren wissenssoziologischen Diskussion skizzieren. ${ }^{1}$ Vorwegzuschicken ist, dass wir keine ExpertInnen der Soziologie sozialer Probleme sind und uns auch nicht anmaßen wollen, dazu fundierte Einschätzungen abzugeben. Zugleich gehen wir von weitreichenden Überlappungen, wenn nicht gar Übereinstimmungen, zwischen zentralen wissenssoziologischen Positionen und Forschungsinteressen sowie den im engeren und weiteren Sinne ,konstruktivistischen' Positionen innerhalb der Soziologie sozialer Probleme aus. Dies gilt unabhängig davon, ob letztere die Untersuchung von Konstruktionsprozessen auf der Ebene von öffentlich-politischen Diskursprozessen verankern oder im konkreten doing social problems der AkteurInnen, Organisationen und Institutionen, die mit problembezogenen Tätigkeiten und der zugrundeliegenden Definitionsarbeit befasst sind. Vor diesem Hintergrund greifen wir nachfolgend Konzepte aus der jüngeren deutschsprachigen Wissenssoziologie - „Problematisierungswissen“ und „Relevanzhorizonte“, „Wissenskulturen“ und „Wissensverhältnisse/Wissenspolitiken“ - auf und verdeutlichen deren mögliche Anregungspotentiale für eine Soziologie sozialer Probleme. Als Ausgangspunkt sei zunächst das Verhältnis von Wissenssoziologie und Soziologie sozialer Probleme beleuchtet.

\section{Wissenssoziologie und Soziologie sozialer Probleme}

Weite Teile der Soziologie sozialer Probleme können seit den 1960er-Jahren als Spielart oder gegenstandsbezogene Anwendung wissenssoziologischer Perspektiven verstanden werden. Das ist keine wissenssoziologische ,Landnahme“, sondern eine einfache Feststellung dazu, wie sich große Bereiche der Soziologie sozialer Probleme in dieser Zeit positioniert haben. 1951 in den USA als Abspaltung der American Sociological Association gegründet, vertrat die Society for the Study of Social Problems von Beginn an eine gesellschaftspolitisch engagierte Position - die Analyse sozialer Probleme sollte unmittelbar zu deren Lösung beitragen (Groenemeyer 2001b, S. 9). Dieses Vorhaben nahm mit der symbolisch-interaktionistischen Wende der Soziologie sozialer Probleme in den 1960er-Jahren eine deutlich wissenssoziologische und später auch analytisch distanziertere Färbung an. Verschiedene Bilanzen der Geschichte der Soziologie sozialer Probleme vor allem in den USA (exemplarisch Adorjan 2019a, b; Best 2016; Dello Buono 2015; Loseke 2015) verweisen denn auch auf die Rezeption und Bedeutung von Peter L. Bergers und Thomas Luckmanns Grundlegung der später im deutschen Sprachraum so bezeichneten ,neuen Wissenssoziologie in ihrem 1966 erschienenen Buch „Die gesellschaftliche Kon-

\footnotetext{
1 Wir bedanken uns bei den Herausgeberinnen des vorliegenden Jubiläumsheftes der Zeitschrift Soziale Probleme für die Einladung zur Beteiligung und gratulieren sehr herzlich zum 30. Jahrgang. Widmen möchten wir diesen Beitrag dem Gedenken an Axel Groenemeyer. Hierzu sei auch auf den von Bereswill et al. (2020) verfassten Nachruf hingewiesen.
} 
struktion der Wirklichkeit" (Berger und Luckmann 1981 [1966]). ${ }^{2}$ Allerdings hält Dello Buono (2015, S. 332) ebenso fest, dass sich im US-amerikanischen Kontext die eigentliche Konsolidierung der Perspektive des social constructionism in der Folge ohne aktiven Dialog mit den erwähnten Autoren bzw. deren Buch vollzogen habe (vgl. bspw. Sarbin und Kitsuse 1994a, b). Wir vermuten, dass dies u.a. den sehr unterschiedlichen historischen Konnotationen des Begriffes Wissenssoziologie in der US-amerikanischen Soziologie und im anglo-amerikanischen Sprachraum geschuldet ist. Wissenssoziologie ist dort einerseits in starkem Maße und im Grunde bis hin zu den jüngeren feministischen und postkolonialen „Standpunkttheorien“ (z. B. Haraway 1988; Harding 2003; Mohanty 2003; Mignolo und Walsh 2018) mit marxistischen Traditionen (z. B. Lukács 1970) und der die Marx-Engelsschen Ausführungen zur Seinsbestimmtheit des menschlichen Bewusstseins generalisierenden Position von Karl Mannheim (1964) verbunden, wird also vor allem mit Situiertheit, Standortbezogenheit oder „Seinsgebundenheit des Denkens“ (Mannheim) assoziiert. Dies ist ausdrücklich nicht mit einem ,radikalen Konstruktivismus ' systemtheoretischer Provenienz und auch nicht mit dem Sozialkonstruktivismus in der Linie von Berger und Luckmann ${ }^{3}$ gleichzusetzen. Andererseits wird Wissenssoziologie bis in die jüngeren Social Studies of Science hauptsächlich - und vor allem im Anschluss an Robert K. Merton (1973) - als Wissenschaftssoziologie verstanden, in der entweder institutionelle Kontexte, Prägungen und Verzerrungen der Herstellung wissenschaftlichen Wissens oder - im Programm des empirischen Konstruktivismus (Knorr Cetina 1989) - die konkrete Arbeit der wissenschaftlichen Wissensherstellung in den Blick genommen wurde.

Während die wissenschaftsbezogene Wissenssoziologie sich offensichtlich für einen anderen Gegenstand interessierte, wäre die Traditionslinie von Marx über Lukács bis Mannheim gewiss eher anschließbar gewesen für eine Analyse des ,moralischen Unternehmertums“ (vgl. Becker 2014a; Gusfield 1983) als Ausdruck spezifischer politischer oder religiöser Positionen, die sich vor dem Hintergrund ihrer jeweiligen Wert- bzw. Moralvorstellungen diagnostizierter ,sozialer Probleme' annehmen. In den Blick gerieten damit ebenso die dahinter stehenden, aus der kapitalistischen Produktionsstruktur resultierenden Herrschaftsinteressen sowie die gesellschaftlichen Macht- und Kontrollverhältnisse - für entsprechende analytische (und in spezifischem Sinne auch durchaus moralkritische) Forschungsinteressen hatte es bereits in der Chicago School etwa bei William I. Thomas oder auch in den Arbeiten von Charles W. Mills Anknüpfungspunkte gegeben. Doch zum einen hätte das die auf die Konstruktionsprozesse bezogenen Fragen des ,Wie?‘ und ,Mit

\footnotetext{
2 Vgl. dazu auch den Überblick von Axel Groenemeyer (2012) über die Entwicklung der Soziologie sozialer Probleme und die aktuelle Bilanzierung der historisch rekonstruierbaren Verflechtungen und Nähen der Positionen von Symbolischem Interaktionismus, der Wissenssoziologie von Berger und Luckmann sowie den Entwicklungen des social constructionism im englischsprachigen Raum bei Nichols (2019); zur Diskussion im deutschsprachigen Kontext der frühen 2000er Jahre vgl. auch Groenemeyer (2001a).

3 Vor allem Thomas Luckmann hat sich vehement von der Bezeichnung (Sozial-)Konstruktivist distanziert (z. B. Luckmann 2010). Dennoch hat sich dieses Etikett zur Bezeichnung des Ansatzes eingebürgert, was wohl vor allem auf Knorr Cetinas (1989) Aufsatz zu den Spielarten des Konstruktivismus zurückzuführen ist. Als dritte Version nennt Knorr Cetina den empirischen Konstruktivismus (auch: Laborkonstruktivismus).
} 
welchen Folgen, für wen?` in eine ganz andere Richtung, nämlich auf das in gesellschaftlichen Erfahrungs- und Interesselagen begründete ,Warum?` eines spezifischen Unternehmertums bzw. Engagements verlagert und die Forschungsinteressen erneut stark verschoben. ${ }^{4}$ Zum anderen bestand mit der pragmatistisch-soziologischen Tradition der Chicago School, den Arbeiten von George Herbert Mead, William I. Thomas, Dorothy Swaine Thomas, Robert Park, Herbert Blumer oder dann auch Howard S. Becker ${ }^{5}$, bereits ein Theorieangebot, dass die individuelle und kollektive Definition von Situationen und Phänomenen sowie die interaktive Prozesshaftigkeit gesellschaftlicher „Diskursuniversen“ (Mead 1934, S. 282) grundlegend thematisiert hatte. ${ }^{6}$ Trotz einer vorübergehend hohen Aufmerksamkeit für die neue Wissenssoziologie von Berger und Luckmann, und trotz auch späterer Hinweise auf deren große Bedeutung (Abbott 2001; Hacking 1999; Groenemeyer 2001b, S. 15 ff.) blieb die längerfristige Resonanz des Werkes im US-amerikanischen und englischen Kontext im allgemeinen sehr begrenzt. ${ }^{7}$ Dies vielleicht auch gerade deswegen, weil Theorieannahmen und Konzepte der interaktionistischen Tradition zu ähnlich gelagert waren und damit eine vergleichbare Argumentationsgrundlage lieferten, ohne den, europäischen philosophischen Ballast “ - etwa die Sozialphänomenologie von Alfred Schütz oder die philosophische Anthropologie von Helmuth Plessner - mitzuschleppen, der sich in der ,gesellschaftlichen Konstruktion der Wirklichkeit“ fand. Rezeptionsasymmetrien haben auch mit wissenschafts- und sprachräumlich unterschiedlichen Ausrichtungen, Verständnisweisen und Resonanzen der jeweiligen Hintergrundphilosophien zu tun.

Stattdessen wurde das elf Jahre später erschienene, ebenfalls klassische Werk von Malcom Spector und John Kitsuse (1977) mit dem Titel „Constructing Social Problems“ zu einer Art Gründungsmanifest einer implizit ,wissenssoziologischen " Perspektive innerhalb der Soziologie sozialer Probleme. Darin wird in Absetzung von moralisch, ethisch und politisch motivierten soziologischen Perspektiven auf soziale Probleme ein analytischer Blick auf die Akteure, Aktivitäten und Folgen des ,claims making“ (Spector und Kitsuse 1977, S. 73-96) entwickelt, der sich vor allem aus dem Symbolischen Interaktionismus und der Ethnomethodologie, letztlich aus dem Umfeld der Chicago School und ihrer Nachfolger u. a. an der Northwestern University in Evanston speiste (Spector 2019).

\footnotetext{
4 Vgl. bspw. das Plädoyer von Dello Buono (2015) für eine kapitalismuskritische Wende der Soziologie sozialer Probleme oder die Beobachtungen von Nichols (2019) zum gegenwärtig bestehenden Außenseitertum des Konstruktivismus im Kontext einer weitreichend politisierten US-amerikanischen Soziologie. Im sozialpolitischen Engagement der 1950er und 1960er-Jahre waren moral- und kulturkritische Stoßrichtungen häufig impliziert. Sie fielen nicht notwendig mit Kapitalismuskritik zusammen.

5 Vgl. etwa Beckers klassische „Außenseiter“-Studie aus dem Jahre 1963 (Becker 2014a) - dort und im weiteren Arbeitszusammenhang von Howard Becker, Herbert Blumer, Joseph Gusfield, Everett Hughes, Anselm Strauss u. a. wurden zentrale und bis heute einflussreiche Begriffe geprägt: moralische Unternehmer, Karrieren, Arenen, doing things together und dergleichen mehr (vgl. auch Becker 1966; Blumer 1971).

${ }^{6}$ Auf die Affinitäten zwischen dem Thomas-Theorem und der jüngeren deutschsprachigen Wissenssoziologie wies bspw. Hitzler (1999) hin.

7 Auch in der Soziologie sozialer Probleme gab und gibt es immer wieder Hinweise auf das Werk von Berger und Luckmann, sowohl im Rahmen der bereits erwähnten Bilanzen und Überblicksbeiträge, wie auch in einzelnen hervorgehobenen Studien (z. B. bei Gusfield 1981).
} 
Soweit wir sehen, entwickelten sich auch in der Folge nur wenige aktive Grenzgängerschaften zwischen der Wissenssoziologie und der Soziologie sozialer Probleme, etwa in den Arbeiten von Michael Schetsche, der seit längerem von der ,Wissenssoziologie sozialer Probleme“" (Schetsche 2000, 2014) spricht. Schetsche zufolge kann ein Phänomen insofern als soziales Problem gefasst werden, wie den darauf bezogenen Diskussionen negative Bewertungen eines Sachverhaltes im Rahmen dominierender Werteordnungen, Annahmen über die Existenz schuldlos Geschädigter sowie über die ethisch gebotene und im Rahmen der bestehenden Ordnung mögliche Behebung dieser Lage zugrunde liegen (Schetsche 2014, S. 49). Sehr weitreichend und seit langem hat insbesondere auch Axel Groenemeyer eine dezidiert wissenssoziologische Ausrichtung der Soziologie sozialer Probleme ausformuliert. Im Zusammenhang einer Rekonstruktion der Positionierungen in der englisch- und deutschsprachigen Soziologie sozialer Probleme unterscheidet er bspw. zwischen der ,soziale[n] Konstruktion als Produktion“ und der „Konstruktion als Interpretation und Definition“ sozialer Probleme (Groenemeyer 2001b, S. 17 ff.): „Tatsächlich ist die Soziologie sozialer Probleme aber durchaus auch als Wissenssoziologie anzulegen, indem nach den Bedingungen für die erfolgreiche (und auch für die nichterfolgreiche) Etablierung von Problemdiskursen in der Öffentlichkeit und Politik gefragt wird“ (ebd., S. 20, mit Verweis u.a. auf Schetsche). Groenemeyer fordert dabei insbesondere eine Berücksichtigung der gesellschaftlichen Kontexte der Konstruktionsprozesse einerseits, des konkreten Problem-(Be-)Arbeitens andererseits: „Selbstverständlich sind soziale Probleme soziale Konstruktionen, allerdings sind sie nur im Ausnahmefall das Ergebnis intendierten Handelns von Akteuren. Problematisierungen sind eingebunden in historisch gewachsene kulturelle, politische und spezifische soziale Kontexte der gesellschaftlichen Entwicklung, die ihnen erst öffentliche und politische Anerkennung sowie Legitimation verleihen oder eben nicht“ (Groenemeyer 2007, S. 6). Daraus resultiert seine Forderung nach einem „Mehrebenenmodell“ zur Analyse von sozialen Problemen als „Institutionalisierung von Problemdiskursen“ (ebd., S. 10). Dieses Modell beinhaltet erstens die Analyse der gesellschaftlichen Kontexte von Problemdiskursen (einschließlich modernisierungstheoretischer Diagnosen), ${ }^{8}$ zweitens die Untersuchung der Formen der Institutionalisierung, und drittens die Ebene des alltäglichen Wirkens organisierter Problembearbeitungen (ebd.).

Ob nun „Wissenssoziologie sozialer Probleme“ oder „,konstruktivistische Soziologie sozialer Probleme“ - betont werden die gesellschaftlichen Problematisierungsund Etikettierungsprozesse in der Konstitution sozialer Probleme. Solche Problematisierungsprozesse kristallisieren - wie man im Anschluss an Joseph Gusfield (1981) formulieren kann - zu spezifischen institutionellen, administrativen und organisatorischen Bearbeitungsweisen, die einen Zusammenhang von Deutungen und Handlungsweisen auf gewisse Zeit relativ verbindlich herstellen. Insoweit können und müssen Zusammenhänge zwischen Interpretationen und ihren handlungspraktischen Konkretisierungen in den Blick genommen werden (Groenemeyer 2012,

\footnotetext{
8 Groenemeyer (2007) verweist hier insbesondere auf die neoinstitutionalistische Weltkultur-Theorie von John Meyer, auf die Bedeutung kultureller Milieus und Organisationen und auf die Nähe zur Beckschen Diagnostik und Analyse von „Risikogesellschaft“ und reflexiver Modernisierung (S. 12).
} 
S. 58-78). Zur Analyse der entsprechenden Prozesse und ihrer Dynamiken sind unterschiedliche Modelle vorgeschlagen worden, die gesellschaftlichen Kontext und Modernisierungsprozesse, „Karriereschritte“, beteiligte ,institutionelle Felder“, die gesellschaftliche Formierung von Diskursen, beteiligte Akteure und deren Diskursstrategien bis hin zur Institutionalisierung der Problembearbeitung in Gestalt von public policies oder organisatorischer Bearbeitung umfassen (ebd., S. 78-104; vgl. Groenemeyer 2001a, 2007, insbes. S. 13 ff.; Groenemeyer et al. 2012; Schetsche 2014, S. 48 ff.).

Viele kritische Diskussionen zum ,Problemkonstruktivismus' in der Soziologie sozialer Probleme (etwa Best 1993) ähneln Debatten, die in Bezug auf die Wissenssoziologie von Berger und Luckmann geführt wurden (vgl. Knoblauch und Pfadenhauer 2019). Beide Richtungen wenden sich gegen den Vorwurf des Relativismus und Idealismus und betonen die mitunter schmerzliche „Realität von Konstruktion“ (Albrecht 2000) - gemeint ist damit nicht nur die Tatsächlichkeit der empirisch beobachtbaren Konstruktionsprozesse, sondern eben auch des ,Konstruierten ' selbst und seiner Folgen: „Konstruktionen [sind] höchst reale Sachverhalte“ (Groenemeyer 2001b, S. 18). ${ }^{9}$ Insofern Soziologie eine Wirklichkeitswissenschaft, also eine empirische Wissenschaft ist, kann sie nicht ohne die Annahme einer beobachtbaren, erforschbaren und insoweit bestehenden Realität sowie entsprechender Gegenstände auskommen. Dies gilt sowohl für einen Konstruktivismus, der auf Wissen, Sprache und soziales Handeln als auch für einen (z. B. ethnomethodologischen) Konstruktivismus, der auf Praktiken und Materialität bezogen ist - die üblichen Abgrenzungen sind für ein tieferes Verständnis der jeweiligen Objektbeziehungen ohnehin wenig hilfreich. ${ }^{10}$

Parallelen gibt es sicherlich auch zwischen der wissenssoziologischen Diskursforschung im Anschluss an Keller (2011) und dem Programm zur Analyse von Problem- bzw. Problematisierungsdiskursen von Groenemeyer (2007). Die Wissenssoziologische Diskursanalyse greift neben dem Anschluss an Berger und Luckmann sowie Alfred Schütz wesentliche Überlegungen von Michel Foucaults Diskurstheorie einschließlich des Dispositivkonzeptes auf und fokussiert die Analyse von gesellschaftlich-historischen Macht/Wissen-Regimen und Problematisierungen (Keller

\footnotetext{
9 Das betrifft auch Kritiken daran, dass der Konstruktionsbegriff zu einem absoluten Relativismus und Idealismus führe. Schon Beckers frühe Studie sah sich der Frage ausgesetzt, ob nun alles eine Frage der Etikettierung oder der Definition sei - „What about murder?“ (Becker 2014b). Gibt es also eine Realität von Phänomenen jenseits des Konstruierten oder ist alles Konstruktion? Solche Fragen durchziehen die entsprechenden Diskussionen bis heute. Becker erklärte kürzlich, dass einige seiner Aussagen in „Outsiders“ (Becker 2014a [1963]) leider falsch gewesen seien. So hätte er bestimmte politische Akteure als „moralische Unternehmer" eingestuft, von denen er jetzt wisse, dass sie aus ganz anderen Motiven heraus gehandelt hätten (Becker 2017, S. $191 \mathrm{ff}$.). Und den Vorwurf, man müsse bei Marihuana nicht erst lernen, die Effekte wahrzunehmen, schließlich sei das doch sofort spürbar, kontert er mit dem ,realistischen “ Hinweis darauf, dass das, was die Musiker der 1950er-Jahre rauchten, eine ganz andere Dosierung und Wirkstoffkonzentration hatte, als die heute im Umlauf befindlichen Cannabissorten (Becker 2014a).

10 Wir schließen uns hier der Aussage von Malcom Spector (2019, S. 178) an, der mit Blick auf die Fragen nach dem Wirklichkeitsstatus von Sachverhalten, die nur durch Deutungs- bzw. Definitionsprozesse zugänglich sind, betont, dass soziologische Fragen und Probleme andere sind als diejenigen der Philosophie und Ontologie, die nach grundsätzlichen Bestimmungen und Existenzweisen fragen: „As sociologists [...] we commit to doing research".
} 
2011, 2019a). Dabei kommen insbesondere einerseits auch die Strukturierungswirkungen von institutionellen und weiteren gesellschaftlichen Kontexten in den Blick, in die das diskursive Handeln gesellschaftlicher Akteure eingebettet ist, andererseits die konkreten dispositiven Interventionen in gesellschaftliche Handlungsfelder (und deren Folgen). ${ }^{11}$ Die umfangreich von Axel Groenemeyer in der Soziologie sozialer Probleme ausgearbeitete Analyse sozialer Probleme begreift diese als Ereignis und Folge öffentlich-politischer Problematisierungen mit institutionellem und handlungspraktischem Niederschlag in Gestalt von spezifischen Zielen, Ressourcen, Techniken und Formen der Problembearbeitung (Groenemeyer 2010, 2012). Es gehe um die „Problematisierung sozialer Probleme als gesellschaftliche Formierung von Diskursen“" (Groenemeyer 2012, S. 82).

Die aus heutiger Sicht insgesamt erstaunlich wenigen Begegnungen zwischen der Soziologie sozialer Probleme und der Wissenssoziologie, insbesondere auch der neueren Wissenssoziologie im deutschsprachigen Raum, sind nicht nur der weiter oben behandelten Entwicklungsgeschichte und Klassikerbildung der Soziologie sozialer Probleme oder einer spezifischen Konnotation von Wissenssoziologie im USamerikanischen und englischsprachigen Kontext zuzuschreiben, sondern umgekehrt auch einer gewissen Ignoranz von Seiten der ,neuen“ Wissenssoziologie, die verschiedene Ursachen hat. Dazu zählt die zwar verspätete, dann aber umso breitere Rezeption der ,gesellschaftlichen Konstruktion“ (Berger und Luckmann) im deutschen Sprachraum - im Wesentlichen eine Folge der Aktivitäten (im Umkreis) von Thomas Luckmann an der Universität Konstanz in den 1980er-Jahren. Die im Vergleich zur Rolle des Werkes in den USA sehr viel größere Bedeutung verdankt sich vielleicht gerade seiner gelungenen theoretisierten Mischung des Anschlusses an europäische Soziologie- und Philosophietraditionen einerseits (etwa Marx, Durkheim, Weber, Schütz, Plessner), an die in der deutschsprachigen Soziologie noch relativ wenig rezipierte Theoriearbeit von George Herbert Mead andererseits - obwohl letzterer im Prinzip seit Mitte der 1960er bis Mitte der 1970er-Jahre in den Positionen des soziologischen Pragmatismus und Symbolischen Interaktionismus mit eingeführt war. Trotz verschiedener Anschlüsse an diese Ansätze in der deutschsprachigen Soziologie - insbesondere in der Kriminalsoziologie, der Soziologie der Sozialarbeit, der sozialen Kontrolle und eben sozialer Probleme - gelang es erst dem Buch von Berger und Luckmann, den Status einer theoretischen Fundierung und eines Grundlagenwerkes zu erlangen. Als mithin die Expansion der ,neuen“ Wissenssoziologie im deutschsprachigen Raum in den 1980er-Jahren mit einem Fokus auf Gattungsanalysen und die Methodologie hermeneutisch-interpretativer Sozialforschung begann, bestand die Sektion Soziologie sozialer Probleme bereits seit 1976 - mit einem starken konstruktivistischen Schwerpunkt im Sinne der zuvor erläuterten Positionen (des Symbolischen Interaktionismus oder etwa Spector \& Kitsuse). Die Umbenen-

\footnotetext{
11 Die Wissenssoziologische Diskursanalyse (Keller 2011) bspw. wurde auch aus der Auseinandersetzung mit den Arbeiten von William Gamson u. a. zur Frame-Analyse von Konstruktionsprozessen im ,public discourse“ heraus entwickelt, schließt jedoch an den stärker wissensanalytisch profilierten Foucaultschen Diskursbegriff an. Soweit wir sehen, wird der Diskursbegriff in der Soziologie sozialer Probleme eher unspezifisch genutzt. Eine Ausnahme stellt bspw. Manabu Akagawa dar, der ebenfalls stärker auf Foucault rekurriert (Akagawa 2015, 2019). Vgl. zur Kritik einer ,diskursvergessenen“ eng gefassten FramingForschung auch Ulrich und Keller (2014).
} 
nung der Sektion „Sprachsoziologie“ der DGS in „Wissenssoziologie“ erfolgte erst im Jahre 2000. Während die Sektion Soziale Probleme seit knapp 35 Jahren besteht und die Zeitschrift Soziale Probleme ihr 30-jähriges Erscheinen feiert, blickt die Sektionsbezeichnung Wissenssoziologie auf zwanzig Jahre zurück. Historisch kann daher wohl davon ausgegangen werden, dass die konstruktivistischen Perspektiven der Soziologie sozialer Probleme bereits eine institutionelle Heimat hatten und keiner neuen bedurften. Hinzu kam, dass die umgegründete Wissenssoziologie mit ihrer starken Prägung durch den Konstanzer Kontext sowie ihre enge Verflechtung mit Sprachsoziologie und Sozialphänomenologie eine deutlich andere inhaltliche Ausrichtung auf Fragen der Methodologie der Interpretation und des Sinnverstehens sowie auf Themenfelder wie z.B. „Alltagswissen“, „kommunikative Gattungen“, „kleine Lebenswelten“ und „Expertenwissen“ annahm - Gegenstände, die für die Soziologie sozialer Probleme wenig relevant waren. Für die letzten beiden Jahrzehnte lässt sich daher wohl von einer gegenseitig gepflegten Nichtwahrnehmung und friedlichen Koexistenz sprechen - mit wenigen, bereits erwähnten Ausnahmen der (beidseitigen) Grenzüberschreitung.

\section{Wissenssoziologische Perspektiven}

Im vorangehenden Abschnitt wurde bereits angedeutet, dass in der heutigen Wissenssoziologie (und seit längerem) verschiedene theoretische Perspektiven nebeneinander bestehen. Sie können an dieser Stelle nicht im Detail diskutiert werden, einige wenige Stichworte müssen genügen. Die an Berger und Luckmann anschließende, wesentlich durch die Grundlagenarbeiten von Alfred Schütz beeinflusste und dann von Hans-Georg Soeffner $(1989,2000)$ geprägte Traditionslinie der „Hermeneutischen Wissenssoziologie“ (Hitzler et al. 1999, 2020) wurde in den letzten Jahren durch diskurstheoretische (Keller 2011), kommunikationstheoretische (Reichertz 2010; Keller et al. 2013; Knoblauch 2016) und subjekttheoretische Positionierungen (Poferl und Schröer 2014) erweitert. Sie bearbeitet derzeit eine Vielzahl von Themenstellungen und konzeptionellen Reflexionen, u. a. auch in Auseinandersetzung mit den Praxistheorien, mit Positionen des Neuen Materialismus (Keller 2017, 2019b) oder der Soziologie der Emotionen (vgl. Dimbath und Pfadenhauer 2021; Poferl und Pfadenhauer 2018; Raab und Keller 2016). Aus den verschiedenen wissenssoziologischen Paradigmen (vgl. Knoblauch 2014; Dimbath und Keller 2017) lassen sich vielleicht nach wie vor ganz unterschiedliche Anregungen für die Soziologie sozialer Probleme gewinnen. So kreisten bspw. die wissenssoziologischen Grundlegungen von Marx und Engels, von Mannheim und bereits auch von Émile Durkheim um die Frage, wie gesellschaftliche Organisationsformen und Institutionen das jeweilige individuelle und kollektive Denken prägen, in unbewussten oder (insbesondere bei Marx und Engels) eben auch in strategisch herrschaftsbezogenen Erscheinungsformen. Bei Durkheim und in anderer Weise bei Mannheim und ihren jeweiligen Nachfolgern implizierte dies die Frage danach, wie soziokulturelle (etwa Religionen) und sozialstrukturelle (etwa Milieus, Klassenlagen) Einbettungen bzw. institutionelle Merkmale dazu beitragen, dass gesellschaftliche Probleme in spezifischer Weise definiert werden und diese Definitionen dann öffentliche Resonanz 
erfahren, bis hin zur Etablierung politisch-instrumenteller Bearbeitungen und der gezielten Erzeugung von moral panics (Cohen 2002 [1972]; Hall et al. 2013 [1978]). Solche Fragestellungen sind thematisch von ungebrochener Aktualität - gerade im Hinblick auf die Klärung von Resonanzbedingungen gesellschaftlicher Problematisierungsprozesse, bspw. in nationalistischen und unterschiedlichsten politisch-ideologisch-fundamentalistischen Bewegungen, wobei die grundsätzliche Fragerichtung nach der Prägung von Wissen und der Definition von Problemen konzeptionell eher erschöpfend bearbeitet scheint. Wir möchten uns im Folgenden daher auf drei Konzepte bzw. Konzeptpaare konzentrieren, die an die Tradition der neueren Wissenssoziologie anschließen und einerseits der Kreativität und Handlungsmächtigkeit der Akteure im Umgang mit Wissensordnungen, andererseits den Institutionalisierungsformen und Strukturierungsprozessen diskursiver Praktiken eine weitaus größere und systematisch einzuholende Bedeutung einräumen. Es handelt sich hierbei um die Begriffe des Problematisierungswissens und der Relevanzhorizonte, der Wissenskulturen und der Wissensverhältnisse/Wissenspolitiken.

\subsection{Problematisierungswissen und Relevanzhorizonte}

Der Begriff des Problematisierungswissens ist in Verbindung mit dem Begriff der sozialen Relevanzhorizonte entwickelt worden und damit von vornherein in einen bestimmten konzeptionellen Verweisungszusammenhang eingebettet (Poferl 2010, 2013, 2016, 2018). Er knüpft an die in der Soziologie sozialer Probleme hinlänglich bekannte Auffassung an, dass soziale Sachverhalte und Gegebenheiten nicht von selbst und ,aus sich heraus' den Charakter eines Problems haben, sondern gesellschaftlich erst definiert und damit zu einem solchen ,gemacht' werden müssen.

In früheren Ausarbeitungen wurde der Begriff wie folgt bestimmt: Problematisierungswissen umfasst sowohl gesellschaftliche als auch subjektive Wissensvorräte und repräsentiert ein spezifisches Gegebenheitswissen. Es zielt darauf ab, dass ein bestimmter Sachverhalt als unakzeptabel/unhaltbar bewertet und damit auch als bearbeitungs- und interventionsbedürftig begriffen wird. Den Prozessen von Problematisierung ist in der Regel die Grundannahme einer Gestaltbarkeit und Veränderbarkeit gesellschaftlicher Verhältnisse eingeschrieben. Erzeugt werden Realitätskonstruktionen und Situationsdefinitionen, die sich gegen andere Wirklichkeitsbeschreibungen richten, vor allem gegen Routinen der Wahrnehmung und gegen Normalitätsannahmen, die - sprichwörtlich - ,kein Problem' in den jeweils angeprangerten Zuständen oder Verhältnissen sehen. Problematisierte Gegenstände stehen für Abweichung, Störung, Irritation - für etwas, was man so nicht (oder nicht mehr) haben will`. Problematisierungen können emanzipatorisch oder disziplinierend sein. Ihre Wirkung lässt sich kaum planen und kontrollieren und ergibt sich auch nicht allein aus Intentionen: Etwas, was ,gut gemeint‘ ist, kann z. B. desaströse Folgen zeitigen. Der Begriff der Problematisierung ist dabei von ,Problemorientierung' insofern abzugrenzen, als er weniger das Vorhandensein von Problemen betont, sondern vielmehr die Definitions- und Konstruktionsleistungen, die das, was als Problem zu gelten hat (und dementsprechend zu bewältigen ist), markieren (Poferl 2013, S. 611).

Jeglichem substantialisierenden oder vermeintlich selbstevidenten Verständnis von Problemhaftigkeit wird damit eine Absage erteilt. Dies heißt ausdrücklich nicht, 
dass bestimmte Zustände oder Prozesse nur dann negative, unangenehme, störende, unerwünschte etc. Eigenschaften haben, wenn sich breitere, etablierte Kreise der Gesellschaft oder überhaupt, irgendjemand ' dafür interessiert. Von einem ,Problem “ zu sprechen, bedeutet aus wissenssoziologischer Sicht, dass diese Eigenschaften erstens in einer Gesellschaft bzw. einer bestimmten abgrenzbaren Sozialwelt, von wem auch immer, als negativ, unangenehm, störend, unerwünscht etc. wahrgenommen und interpretiert werden. Die Problematisierung richtet sich zweitens auf soziale Sachverhalte und Gegebenheiten, die als gestaltbar und damit als - im Prinzip veränderbar gelten. Damit kommen zwei konstitutive, jedoch oftmals übersehene Komponenten des Gegenstandsbereichs in den Blick: Subjektivität, Individualität und deren Geschichtlichkeit zum einen, das Moment der Selbstgestaltung von Moderne zum anderen. Subjekt- und gesellschaftstheoretische Überlegungen gehen in den hier vorgeschlagenen Konzeptionen insofern Hand in Hand.

Aus subjekttheoretischer Sicht setzt der Begriff des Problematisierungswissens spezifische lebens-, alltags- und sozialweltliche Relevanzen voraus. Dies ist analytisch folgenreich. Die Aufmerksamkeit wird auf die Relevanzsetzung, auf die Entstehung von Relevanzen und deren Wirkungen, mit anderen Worten: auf Prozesse der Zuschreibung von Bedeutsamkeit, gelenkt. Daran knüpft explizit der ergänzende Begriff der sozialen Relevanzhorizonte an. Die Akzentsetzung ist hierbei eine andere als im Schützschen Begriff der Relevanzsysteme (Schütz und Luckmann 1979; Schütz 1982). Von Relevanzhorizonten zu sprechen, betont das Offene, Vage, Instabile und Uneindeutige, das sich mit entsprechenden Relevanzen nicht notwendigerweise, aber durchaus auch verbinden kann. Des Weiteren geht es um Deutungs-, Wissens- und Denkräume, die sich auftun, ohne bereits die Klarheit, Struktur und Stabilität eines ,Systems` zu haben.

Eine solche Bedeutsamkeit entsteht nicht allein und nicht vorrangig aus vorgegebenen Wissensordnungen, Institutionen und Deutungsschemata - so wichtig diese sind. Die auf spezifischen Relevanzhorizonten aufbauenden und gegebenenfalls in neue, veränderte Relevanzhorizonte mündenden Formen von Problematisierungswissen haben ihre Grundlage im subjektiven, individuellen Erleben und Erfahren sozialer Wirklichkeit, das sich einerseits aus kollektiven Wissensbeständen speist, andererseits (mehr oder minder) erheblich davon abweichen und diametral gegenläufig gestaltet sein kann. Nur so lässt sich der schlichte, gleichwohl verbreitete Umstand erklären, dass eine Person etwas ,als Problem' empfinden kann, was andere - wortwörtlich - nicht tangiert. Die Differenz und Divergenz von Erleben und Erfahrung (die ganz unterschiedliche Gründe haben kann) spielt hierbei eine zentrale, problemkonstitutive Rolle. Die Geschichtlichkeit und kulturelle Formierung von Subjektivität wird in einem solchen, auf das Individuum und dessen Weltverhältnisse ausgerichteten Konzept nicht unterschlagen, im Gegenteil. Für einen nicht naiven und wissenssoziologisch informierten Zugang sind historische und kulturelle Prozesse des Wandels z. B. von Gefühlen (was wird unter welchen historischen Bedingungen als Scham, Demütigung oder Grausamkeit empfunden? Wem gilt Empathie?) höchst aufschlussreich, um die gesellschaftlichen Hintergründe von Erlebensund Erfahrungsprozessen auszuleuchten. Sie ersetzen die Frage nach der Bedeutung von Subjektivität nicht, sondern vertiefen das Verständnis. 
Gesellschaftstheoretisch sind beide Konzepte - Problematisierungswissen und Relevanzhorizonte - durch die von Ulrich Beck und anderen entwickelte Theorie reflexiver Modernisierung (Beck et al. 1996; Beck und Lau 2005) geprägt. Ausgehend von Funktions- und Legitimationskrisen der westlichen, industriegesellschaftlichen Moderne stellt diese Theorie das - die ,Moderne“ im Unterschied zur ,Vormoderne' auszeichnende - Merkmal der gesellschaftlichen Selbstgestaltung in den Vordergrund. Es löst die Unterwerfung unter eine Natur- oder göttliche Ordnung zumindest institutionell und auf der Ebene der Selbstbeschreibung moderner Gesellschaften ab, was wesentlich auch Fragen der Identität, des Selbst betrifft (vgl. Taylor 1996). Die Theorie reflexiver Modernisierung betont darüber hinausgehend die Dynamiken einer gleichsam entfesselten und die etablierten Institutionen letztlich umstürzenden Selbstaufhebung - eine „Modernisierung der Moderne“ (Beck et al. 2001) -, die nicht nur einzelne ,problematische“ Erscheinungsformen oder Teilfragen, sondern die Moderne in ihrer herkömmlichen Gestalt zum „Problem“ werden lassen: „Die westliche Moderne wird sich selbst zum Thema und Problem; ihre Basisprinzipien, Grundunterscheidungen und Schlüsselinstitutionen lösen sich im Zuge radikalisierter Modernisierung von innen her auf; das Projekt der Moderne muss neu verhandelt, revidiert, restrukturiert werden“ (Beck et al. 2001, S. 11).

Eine solche Beschreibung von Strukturentwicklungen und damit verbundenen Herausforderungen lässt gängige, gleichsam ,systemimmanente', Verständnisse von Problemdefinitionen und Problembearbeitungen hinter sich. Die Theorie reflexiver Modernisierung ist kein dezidiert wissenssoziologischer Ansatz, wenngleich sie eine Fülle von (de facto) wissenssoziologischen Aussagen und Argumenten enthält. Sie ist auch nicht mit einer Soziologie sozialer Probleme zu verwechseln. Als Gesellschaftstheorie macht sie jedoch darauf aufmerksam, dass jede die Grundfesten und Grundmuster moderner Gesellschaften berührende Problematisierung zu institutionellen und sozialweltlichen ,Grundsatzfragen' führen kann und entsprechend Sprengkraft enthält. Auch dies ist für die Begriffe des Problematisierungswissens und der Relevanzhorizonte zentral. Eine Befassung mit Problematisierungswissen und damit verbundenen, virulent werdenden oder sich neu eröffnenden Relevanzhorizonten muss nun keineswegs so weit gehen, stets und in jedem Fall die Frage des gesellschaftlichen Ganzen bzw. der Strukturzusammenhänge einer sich wandelnden Moderne in den Blick zu nehmen. Sie sollte es aber tun, wenn sie nicht Gefahr laufen will, mit verkürzten oder gar den jeweiligen vorherrschenden Wissensordnungen, Deutungskontexten und Normalitätsannahmen unterworfenen Blickwinkeln $\mathrm{zu}$ arbeiten. Ein subjekt- und gesellschaftstheoretisch interessierter Gebrauch beider Konzepte kommt nicht ohne einen solchen weit aufgespannten Theorierahmen aus. Sie beziehen vielmehr gerade daraus den Anspruch, Potentiale der gesellschaftlichen Transformation oder der „Metamorphose“ (Beck 2017) der Welt, d.h. des Wandels von Gestalt und Gehalt, Formen und Zusammensetzungen, über tradierte, westlich-industriegesellschaftliche Ausprägungen hinaus zu erfassen. Beispiele hierfür sind etwa die Entwicklung globaler Problemwahrnehmungen und globaler Solidaritäten (am Beispiel globaler Armut Poferl 2010, 2013; Poferl und Walter 2013) sowie die Entstehung und Verbreitung einer transnationalen Wissensfigur des Menschenrechts- und Menschenwürdesubjekts (Poferl 2018; unter Berücksichtigung multipler Geschlechterkulturen Poferl 2020). Daran kann dann auch die Frage nach 
Problematisierungsprozessen und Relevanzhorizonten im Kontext vielfältiger, unterschiedlicher und ungleicher pluraler Modernen (vgl. Eisenstadt 2000; Beck und Grande 2010; Winkel und Poferl 2020) anschließen.

Im Kern zielen beide Begriffe auf die Schlüsselfrage, was wen wie warum etwas , angeht', und auf die Antworten, die darauf - sei es auf der Makro-, Meso- oder Mikroebene sozialer Wirklichkeit - gefunden werden müssen. Die damit verknüpften Prozesse z. B. der Entwicklung von ,vocabularies of motives“ (Mills 1940) und der Ausbildung von Rechtfertigungen für soziales und politisches Handeln (vgl. Boltanski 1999; Boltanski und Thévenot 2007) sind dabei nicht vorhersehbar und durch und durch kontingent. Weder ein einseitig auf Disziplinierungs- und Kontrollmacht beschränkter Ansatz (wie man ihn sowohl aus marxistischen als auch aus poststrukturalistischen Theorien ableiten könnte) noch eine unreflektierte Verdoppelung gesellschaftlich vorfindlicher moralischer und politischer Problemauffassungen wird dem gerecht.

\subsection{Wissenskulturen und soziologische Erkenntnisbildung}

Es gibt vermutlich nicht viele sozial- und kulturwissenschaftliche Begrifflichkeiten, die in wenigen Jahren (d.h. in wissenschaftlich relativ kurzer Zeit) eine so starke Verbreitung erfahren haben, wie dies beim Begriff der Wissenskulturen der Fall ist. Mit sozialen Problemen hat der Terminus auf den ersten Blick wenig zu tun. Seine Grundlagen liegen führend in den Geistes- und Kulturwissenschaften, der Geschichtswissenschaft, der historischen Soziologie sowie der Wissenschaftsforschung, wobei es gegenwärtig eine große Bandbreite an Verwendungsweisen sowie zahlreiche Abhandlungen und Studien in verschiedenen Wissenschaftsdisziplinen (vgl. zum Überblick Poferl und Keller 2018).

In der Soziologie, genauer: der Wissenschaftssoziologie, wurde der Begriff Wissenskulturen unter dem Originaltitel „epistemic cultures“ wegweisend von Karin Knorr Cetina in einer vergleichenden Studie zur Molekularbiologie und zur Hochenergiephysik (Knorr Cetina 2002) eingesetzt. In der von ihr entwickelten, viel zitierten Begriffsbestimmung umfassen Wissenskulturen demnach ,diejenigen Praktiken, Mechanismen und Prinzipien, die, gebunden durch Verwandtschaft, Notwendigkeit und historische Koinzidenz, in einem Wissensgebiet bestimmen, wie wir wissen, was wir wissen“. Die Aufmerksamkeit gilt den ,Strategien und Prinzipien, die auf die Erzeugung von ,Wahrheit" oder äquivalente Erkenntnisziele gerichtet sind" (Knorr Cetina 2002, S. 11; Hervorh. im Original). Gleichwohl ist die grundlegende kulturelle Prägung und Gestaltung der Hervorbringung wissenschaftlichen Wissen auch für soziologisches Wissen von Bedeutung. Dies gilt für jedes Teilgebiet der Soziologie und für die Soziologie sozialer Probleme vielleicht sogar in besonderer Weise. Darauf wird zurückzukommen sein.

In unseren eigenen Forschungen haben wir den Begriff der Wissenskulturen auf die Entwicklung von Methoden im Feld der qualitativen Sozialforschung im deutschfranzösischen Vergleich angewandt. Dies muss hier inhaltlich nicht weiter ausgeführt werden. Die Forschung hat uns allerdings zu einer - teils diskursanalytischen, teils pragmatistisch-handlungstheoretischen - Weiterentwicklung des Begriffs der Wis- 
senskulturen veranlasst. ${ }^{12}$ Aus einer wissenssoziologischen Perspektive schlagen wir vor, wissenschaftliches Arbeiten als Grundlage und Ausdruck der wissenschaftlichen Konstruktion von Wirklichkeit zu betrachten und darin zum einen sowohl den institutionell mehr oder minder stabilisierten diskursiven Strukturierungen als auch zum anderen den kontextuell situierten Erkenntnisorientierungen wissenschaftlichen Handelns nachzugehen. In der empirischen Praxis wissenschaftlichen Arbeitens sind beide Ebenen bzw. Dimensionen miteinander verschränkt, insofern handelt sich um eine analytische Unterscheidung. Sie erscheint uns allerdings hilfreich, um die - empirisch mögliche und gegenständlich sogar unabweisbare - Differenz zwischen diskursiver Strukturierung und Handlungsorientierung, und das heißt letztlich auch: das Wechselspiel von Zwängen und Freiheitsgraden von Wissenskulturen, zu erfassen.

Hierbei gehen wir von zwei grundlegenden Annahmen aus: (1) Diskursive Strukturierungen - mit anderen Worten: Konventionen und Legitimationen wissenschaftlichen Arbeitens - sind dem wissenschaftlichen Handeln nicht einfach vorgelagert, sondern inhärent. Sie begrenzen und eröffnen den Möglichkeitsraum wissenschaftlichen Handelns. Aufgrund seiner disziplinären Verankerung sind dem wissenschaftlichen Handeln, konzipiert als sinnhaftes Handeln, diskursive Vorgaben gleichsam eingeschrieben. (2) Wissenschaftlich sinnhaftes Handeln enthält potentiell jedoch immer auch Formen des ,Eigensinns “ und anarchistische Momente, die dem diskursiv ,Sagbaren“ und ,Zeigbaren“ entgehen oder zuwiderlaufen und gerade darin Kreativität entfalten. Man muss dies nicht dramatisieren. Geradezu typisch erscheint uns vielmehr ein Anarchismus, der seine Wurzeln in der jedem wissenschaftlichen Handeln konstitutiv zu eigenen Befassung mit Gegenständen der natürlichen und sozialen Welt sowie dem Bemühen um ein empirisches und analytisches Verstehen dieser hat - zumindest dann, wenn es sich von der Idee der Gegenstandsangemessenheit und dem darauf abgestellten Bemühen um eine theoretische Durchdringung des wissenschaftlichen Vorgehens leiten lässt. Darin kommt die grundlegende Problemorientierung wissenschaftlichen Handelns als erkenntnisproblemlösendem Handeln zum Tragen (Poferl und Keller 2018, S. $30 \mathrm{ff}$.).

Die wissenschaftliche Analyse sozialer Probleme ist mit spezifischen gegenständlichen Herausforderungen konfrontiert: Beforscht werden Probleme, die im gesellschaftlichen Kontext, wie auch immer, schon als ,soziale Probleme' definiert worden sind und somit eine (mehr oder minder) verbreitete Problemthematisierung sowie gegebenenfalls eine institutionalisierte Problembearbeitung erfahren haben. Dass die Gegenstände der sozialen Welt sich durch ein bereits vorhandenes soziales SelbstVerstehen auszeichnen, ist für sich genommen nicht weiter überraschend. Vielmehr begründet dies ein zentrales Prinzip und Merkmal der „doppelten Hermeneutik“ (Giddens 1984 [1976]), die dem Prozess des „Verstehen des Verstehens“ (Hitzler 1993 ) konstitutiv abverlangt ist. Gemeint ist damit, dass soziologisches, also wissenschaftliches, Verstehen das soziale Selbst-Verstehen zur Kenntnis zu nehmen und gegenständlich zu berücksichtigen hat. Dies bedeutet aber auch, mit der ,Eigensinnigkeit' und hochgradigen sozialen Reaktivität des beforschten Feldes, d.h. der sozialen Welt in ihren je unterschiedlichen Erscheinungsformen, rechnen zu müssen.

12 Wir greifen im Folgenden teilweise auf Ausführungen von Poferl und Keller (2018) zurück, die wir hier übernehmen. 
Im Unterschied zu bspw. unbelebten Gegenständen nehmen Personen, Gruppen und menschliche Gemeinschaften die Tatsache des Beforscht-Werdens nicht passiv und auch nicht immer erfreut zur Kenntnis. Die Soziologie sozialer Probleme ist darüber hinaus womöglich mit einer besonderen Widerständigkeit des Feldes konfrontiert, das sowohl seine eigenen Problemdefinitionen und Problemlösungen als auch ausgeprägte Erwartungen an ,von außen' kommende wissenschaftliche Forschung hat sei es z. B. die Hoffnung auf rasch umsetzbare anwendungsorientierte Hilfestellung, sei es der Wunsch nach Aufmerksamkeit und Verständnis der jeweiligen Problemlagen, Szenen und Milieus oder aber umgekehrt das Bedürfnis, eher nicht beforscht und ,in Ruhe' gelassen zu werden.

Die produktive Verflechtung der (im besten Sinne) erfinderischen, kreativen Elemente wissenschaftlicher Arbeit mit feldspezifischen Bedingungen, eine Mischung aus Sensibilität, Phantasie und analytischer Distanz kann vor einem solchen Hintergrund zu ungeahnten Einsichten und Höhenflügen der Erkenntnisbildung führen. Hingegen kann die Unterwerfung unter Begriffsregime und einen vermeintlich vorgeschriebenen Methodenkanon, die Fixierung auf womöglich falsch formulierte Forschungsfragen oder ein nicht gelingender, (offen oder verdeckt) scheiternder Feldzugang in (wechselseitig) unauflösbares Nicht-Verstehen münden. Vor allem die Methodologie der interpretativen und ethnographischen Sozialforschung hat sich zentral mit solchen Problemstellungen befasst. Qualitative Forschungstraditionen nicht nur, aber vor allem der (US-amerikanischen) Soziologie sozialer Probleme, wie sie im ersten Abschnitt dieses Beitrags genannt sind und ebenso aktuelle Analysen zeugen z. B. von den Anstrengungen, Beziehungen zum Feld und Wege ins Feld (vgl. Poferl und Reichertz 2015) aufzubauen - von den Mühen der gegenstandsadäquaten und zu neuem soziologischem Wissen führenden Erkenntnisbildung ganz zu schweigen.

Von soziologischen Wissenskulturen zu sprechen, impliziert weder ein essentialistisches noch statisches noch raum-zeitlich geschlossenes Verständnis von ,Kultur sondern setzt - darin Kulturbegriffen von Max Weber und Clifford Geertz folgend - an Sinn- und Bedeutungszuschreibungen wissenschaftlichen Handelns sowie der darin eingelassenen Interpretativität an. Es geht um historisch hervorgebrachte Symbolisierungs-, Diskurs- und Praxisfelder, welche spezifische Formen und Muster des wissenschaftlichen Deutens und Handelns ausbilden und vorübergehend stabilisieren. Soziologische Wissenskulturen beinhalten zugleich aber auch ein gewisses $\mathrm{Ma}$ an Freiheit von disziplinären Vorgaben, ohne das die Lösung wissenschaftlicher Erkenntnisprobleme nicht möglich ist. Hierbei geht es um die Freiheit, die Gegenstände so zu erfassen, wie diese es erfordern. Dies ist kein naiver Naturalismus, sondern, wenn überhaupt, ein ,Naturalismus', der sich aus der Weltzugewandtheit wissenschaftlichen Arbeitens speist, die wiederum nur um den Preis des Empirieverlustes aufgelöst werden kann.

Vor welche Herausforderungen sind soziologische Wissenskulturen heute gestellt - auch über den rein binnenwissenschaftlichen Horizont hinaus? Wiederholt hinzuweisen wäre hier unseres Erachtens erstens auf die Verwissenschaftlichung und vor allem auch die beobachtbare Soziologisierung von Gesellschaft, die die Grenzen zwischen Wissenschaft und Nicht-Wissenschaft teilweise verschwimmen lässt. Dem stehen zweitens seit den 1960er, 1970er-Jahren, nicht zuletzt befördert durch den Positivismusstreit und seine Nachwirkungen, Prozesse der Politisierung von Wissen- 
schaft gegenüber. Das Webersche Postulat der „Wertfreiheit“ wird dadurch nicht gänzlich in Frage gestellt, es geht primär um den wissenschaftlichen Beitrag zur politischen Gestaltung von Gesellschaft; Selbstpositionierungen und Selbstetikettierungen als ,kritische Wissenschaft" sowie der Bezug auf Intervention im Unterschied zu Affirmation sind Beispiele hierfür. Kontrastierende Auffassungen begreifen Kritik dagegen als inhärentes Moment der Soziologie, das sich aus der Beobachtung ,zweiter Ordnung“ (Niklas Luhmann) bzw. den Konstruktionen ,zweiten Grades“ (Alfred Schütz) ergibt und gerade nicht der Verortung in der politischen Praxis bedarf. $\mathrm{Zu}$ nennen sind drittens auch verschiedenste neuere Varianten der erkenntnistheoretischen und Methodenkritik, die an Standards und Konventionen soziologischer Erkenntnisproduktion ansetzen. Die feministische Forschung - darauf wurde zuvor schon hingewiesen - hat seit jeher die Befangenheiten androzentrischer Sichtweisen problematisiert. Aus post- und dekolonialer Perspektive werden seit einiger Zeit südliche Epistemologien (de Sousa Santos 2014) und indigene Methodologien (Kovach 2009) diskutiert, die auf ihre Weise an der Frage des ,Was' und ,Wie“ wissenschaftlichen Wissens ansetzen. Innerhalb der qualitativen Sozialforschung werden Vorschläge zur Entwicklung einer ,post-qualitative research“ (vgl. Lather und St. Pierre 2013) formuliert, die sich der Einengung auf gängige Zugänge und Verfahrensweisen entzieht und möglichst differente Quellen sowie (z. B. wissenschaftliche, künstlerische, performative) Formen der Erschließung von und des Erzählens über die Welt nutzen will. Hinzu kommen viertens jüngste - und so kaum vorhersehbare Tendenzen der politischen, gesellschaftlichen und wissenschaftlichen Polarisierung und Fundamentalisierung, die von der (wissenschaftsexternen) Diskreditierung wissenschaftlichen Wissens im Zuge neoautoritärer Strömungen und Regime einerseits bis hin zur (wissenschaftsinternen) Aufrüstung quantifizierend-kausalanalytischer Ansätze andererseits reichen. Wir sind geneigt, in all diesen - vielfältigen und widersprüchlichen, teils uneindeutigen, teils recht eindeutigen - Entwicklungen den Ausdruck einer Transformation von Moderne und moderner Wissenschaft zu sehen, die, aus gegenständlichen Gründen naheliegend, gerade die Soziologie in ihrem Kern - der Analyse gesellschaftlicher Wirklichkeit - tangieren (Poferl und Keller 2018, S. 32 und S. 35 f.).

\subsection{Wissensverhältnisse und Wissenspolitiken}

Die Begriffe der Wissensverhältnisse und der Wissenspolitiken werden hier im Sinne der Wissenssoziologischen Diskursanalyse (WDA; vgl. Keller 2011) verstanden. Diskurse sind institutionell-organisatorisch regulierte Praktiken des Zeichengebrauchs. In und vermittels von Diskursen wird von gesellschaftlichen Akteuren im Sprach- bzw. Symbolgebrauch die soziokulturelle Bedeutung und Faktizität physikalischer und sozialer Realitäten konstituiert. Die allgemeine Passung dieser Perspektive zur konstruktivistischen Soziologie sozialer Probleme ist nicht zufällig. Kernüberlegungen der WDA wurden im Anschluss an diese Forschungsperspektiven entwickelt (Keller 2011, S. 73-82).

Das Konzept der Wissensverhältnisse impliziert eine wissenssoziologische Erweiterung des Begriffs der „Definitionsverhältnisse“, den Ulrich Beck im Rahmen 
seiner Risikosoziologie vorgeschlagen hat. ${ }^{13}$ Beck adressiert damit die Akteure und Mechanismen, die zum Einsatz kommen, um das Risikopotential von Stoffexpositionen, technologischen Systemen, Gefährdungslagen usw. zu bestimmen. Der Begriff impliziert unterschiedlich verteilte, institutionell-diskursiv stabilisierte oder in Frage gestellte Möglichkeiten der Verfügung über Wissensressourcen, damit auch Machtgefälle, Asymmetrien und Konfliktlagen. Die Bestimmung von Grenzwerten bspw. ist keine Festlegung einer objektiven Tatsache, sondern Ergebnis einer (sub-)politischen Aushandlung, Wissenserzeugung, Abwägung und Machtausübung (vgl. Beck 1988). Wissensverhältnisse beinhalten sehr unterschiedliche Formen der Evidenzbildung und darauf beruhende Artikulationen des ,Faktischen', die sich auf ebenso unterschiedliche Wissensformen stützen und mit je spezifischen Wertungen einhergehen.

Wenn Adorjan (2019, S. 162) festhält, ,claimsmaking discourses are fundamentally moral discourses", so weist der Begriff der Wissenspolitiken auf die aktiv von gesellschaftlichen Akteuren eingebrachten Aussagebestandteile hin, welche die ,Tatsächlichkeit" und Existenzweise eines Phänomens mit ,Evidenz versehen oder sie herausfordern - einer Evidenz, die ganz unterschiedliche Formen annehmen kann. Solche Aussagen sind, wie u. a. schon Berger und Luckmann (und in gewissem Sinne auch Max Weber) betonten, nahezu immer Verflechtungen von Wertungen und Tatsachen. Ob ein Stoff ungefährlich ist, ergibt sich nicht zwangsläufig aus seiner ,Tatsächlichkeit‘. Ob das Glas Wasser halb voll oder halb leer ist, ergibt sich nicht aus seinem ,Faktum‘. Ob ein soziales Problem ,kritisch' ist, ist keine Frage seiner ,Messung', sondern eine der Bewertung von ,Messgrößen“ und ,Realitätsprüfungen'. Wissenspolitiken sind deswegen wertvolle Interventionen in gesellschaftliche Problematisierungskontexte und die dortigen Wissens- und Regulierungsverhältnisse. In einem laufenden Projekt zur gegenwärtigen Regulierung von Prostitution in Deutschland beobachten wir bspw. arenenspezifische Wissenspolitiken in Gestalt von medizinisch-psychologischen Expertisen, kriminalpolizeilichen Erfahrungsberichten, des berufsverbandlichen Engagements und anderes mehr, die ganz unterschiedliche Formen der ,Beweisführung ‘ - etwa eine Generalisierung des beispielhaften Einzelfalles, eine Politik der großen Zahlen, statistische Erfassungen und anderes mehr - vornehmen. In diesem Zusammenhang von Diskursen zu sprechen, impliziert, dass die gegebenen und die möglichen Formen der Aussageproduktion aus spezifischen historischen Formationen von Aussageweisen resultieren.

Der Diskursbegriff verweist auf die Musterförmigkeit der Bedeutungserzeugung, auf ihre Regeln und Grenzen, auf das, was sagbar, und das, was nicht sagbar erscheint - Diskurse sind spezifisch regulierte gesellschaftliche Formen der Wissensprozessierung. Der Begriff der Wissenspolitiken bezieht sich also auf die kommunikativen Handlungen und Strategien von gesellschaftlichen Akteuren, spezifische Wissensverhältnisse zu stabilisieren, anzugreifen, zu verteidigen und zu transformieren. Es geht um Wirklichkeitspolitiken in unterschiedlichsten gesellschaftlichen Feldern, etwa auch im Kontext von Religionen und politischen Ideologien bzw.

\footnotetext{
13 Beck bildet den Begriff der Definitions[macht]verhältnisse in Analogie zum Marxschen Konzept der Produktionsverhältnisse, ohne allerdings die marxistische Gesamtanalyse zu übernehmen. Damit ähnelt der Becksche Begriff eher dem Foucaultschen Konzept der Macht/Wissen-Regime.
} 
politischen Mobilisierungen. Wenn von Wissensverhältnissen und Wissenspolitiken gesprochen wird, dann impliziert das Einrichtungen (Artefakte, Verfahren, Handlungsweisen, Infrastrukturen) unterschiedlichster Art - Dispositive als Infrastrukturen der Diskursproduktion und als Infrastrukturen der Bearbeitung von definierten ,kollektiven ' Handlungsproblemen. In Dispositiven sind Praktiken, Objekte (Materialitäten), Akteure, ,Gesagtes und Nicht Gesagtes ' in mehr oder weniger komplexen Gemengelagen verflochten. Im hier gemeinten Sinne sind Infrastrukturen historische Resultate ganz unterschiedlicher aufeinander bezogener, ineinander verketteter Handlungen. Sie liegen zum einen als Ressourcen unmittelbar der Prozessierung spezifischer Diskurse zugrunde. Sie kommen zum anderen als eingreifende Artefakte, Texte und Handlungen ganz unterschiedlicher Art in den Blick - als hybride Bearbeitungsformen identifizierter „Notstände“ (Michel Foucault) mit häufig ungeahnten, ungesehenen, ungewollten Folgen.

\section{Aktuelle Herausforderungen für eine wissenssoziologische Betrachtung sozialer Probleme}

Unser Anliegen im vorliegenden Beitrag ist nicht, eine erneute „Wissenssoziologie sozialer Probleme" (Schetsche 2014) zu entwerfen. Vielmehr geht es uns darum, über einige ausgewählte konzeptionelle Vorschläge den Raum für weitere, wechselseitig fruchtbare Begegnungen zwischen Wissenssoziologie und Soziologie sozialer Probleme zu eröffnen. Etliche der nachfolgend noch einmal rekapitulierten Beobachtungen gelten daher ebenso sehr für die Wissenssoziologe wie vielleicht auch für die Soziologie sozialer Probleme. Eher stichpunktartig und die vorherigen Überlegungen zusammenfassend seien abschließend drei Aspekte aufgegriffen, die, ausgehend von unserer wissenssoziologischen Betrachtung, die theoretische und empirische Analyse sozialer Probleme künftig herausfordern könnten.

1. Die gesellschaftliche Entwicklung der Gegenwart scheint erstens mehr denn je in eine gesellschaftliche Praxis zu münden, die einem Experiment mit sich selbst gleichkommt. Dieser Gedanke ist nicht neu. Er wurde einerseits im Hinblick auf technologische und umweltbezogene Risiken sowie darauf bezogene Forschungsund Gestaltungsprozesse geprägt und systematisch im Begriff der „Realexperimente" von Wolfgang Krohn (2007) ausbuchstabiert. Diese Idee wurde vor allem in den gegenwartsdiagnostischen Debatten um die Entstehung der ,Wissensgesellschaft ' weiterverfolgt. Über den wissenschafts-, technik- und umweltsoziologischen Kontext hinaus und unabhängig davon ist der Gedanke einer „Gesellschaft im Selbstversuch“ (Poferl 1999) andererseits aber auch in einem weiteren, die Analyse der sozialen Welt und gesellschaftlicher Modernisierungsprozesse schlechthin umfassenden Sinne entwickelt worden. Den Hintergrund bildet die im vorliegenden Beitrag mehrfach erwähnte Theorie reflexiver Modernisierung, die für eine nicht nur empirisch ausgerichtete, sondern auch gesellschaftstheoretische Wendung der Wissenssoziologie aus unserer Sicht richtungweisend ist. Die Argumentationsfigur einer mit sich experimentierenden Gesellschaft besagt, dass zunehmend weniger von etablierten, institutionellen Gepflogenheiten und Deu- 
tungskonventionen, von Routinen des Denkens und Handelns in den ausgetretenen Pfaden des (vermeintlich) Immer-schon-so-Seienden, des Bekannten und Bewährten ausgegangen werden kann. Dies gilt im Hinblick auf die soziale Ordnung, auf soziale Prozesse, auf gesellschaftliche Verhältnisse und deren Wandel selbst ebenso wie für die Soziologie, die mit der Untersuchung all dessen befasst ist. Das unhinterfragte, blinde Festhalten an Altem lässt wenig Raum für die Entstehung von Neuem - eine Binsenweisheit, die gleichwohl höchst reale Folgen hat. Für eine wissenssoziologisch ausgerichtete Analyse sozialer Probleme bedeutet dies zum Beispiel, sich von herkömmlichen Problembetrachtungen und analytischen Zugängen (auch) zu lösen und die Klärung der Frage, was über welche Probleme überhaupt bekannt sein kann und was sich dem ,Auge des wissenschaftlichen Betrachters/der wissenschaftlichen Betrachterin` verschließt, mehr denn je ernst zu nehmen. Hierbei geht es ausdrücklich nicht darum, in einen Wettstreit der jeweiligen Positionierungen, Situierungen und Erfahrungsprivilegien zu treten und auf dem jeweils vermuteten ,Unvermögen“ des jeweils ,Anderen“, der/die man nicht selbst ist, zu insistieren - kaum etwas ist der sozial- und kulturwissenschaftlichen Erkenntnis und Inspiration weniger förderlich, als die Selbstbeschränkung auf den je eigenen Horizont (dies ließe sich z. B. an identitätspolitischen Aufladungen auch des wissenschaftlichen Diskurses trefflich studieren). Wissenschaftliches Interesse für die Entstehung von Neuem bedeutet gerade umgekehrt, sich dem Nicht-Bekannten, Nicht-Gewussten, Nicht-Erahnten auszusetzen und die Erzeugung von Wissen, die Erkenntnisbildung, dadurch zu bereichern, d.h. produktiv zu irritieren. Die Befassung etwa mit Problematisierungswissen und Relevanzhorizonten auch über den nationalen Blickwinkel hinaus, wie sie vorangehend in Abschn. 3.1 angesprochen worden ist, kann davon profitieren.

2. Es sind zweitens nicht allein, aber vermutlich in gesteigertem Maße Prozesse der Globalisierung und Transnationalisierung, die gegenwärtig in eine kosmopolitische Situation münden und die Entwicklung eines „,kosmopolitischen Blick(s)“ (Beck 2004) sowohl in der sozialen Welt als auch in der Wissenschaft erfordern. Die kosmopolitisierte Gesellschaft entzieht sich oder untergräbt die scheinbar selbstverständlichen, institutionalisierten Problemdiskurse und Problembearbeitungen der ,einfachen Moderne“ (Beck 1986) - mit noch nicht absehbaren Folgen. Die Begrifflichkeit des Kosmopolitischen sollte nicht oberflächlich verwendet werden, es geht hierbei keineswegs um sozialstrukturelle Merkmale eines irgendwie weltbürgerlich versierten Milieus, einer Elite oder Szene, der es möglich ist, halbwegs sicher den Globus zu bereisen, souverän in mehreren Sprachen zu parlieren etc. Eine kosmopolitische Situation besteht im wissenschaftlich relevanten Sinne darin, sich genau dieser Erosion des ,Bekannten “ und seiner unabweisbaren Verflochtenheit mit der ,Andersheit', der Differenz, dem Nicht-Bekannten, NichtGewussten, Nicht-Erahnten oder schlicht Exkludierten auszusetzen (vgl. z. B. Poferl 2015, 2020). Das heißt auch, sich die ,andere', der bisherigen wissenschaftlichen Betrachtung womöglich abgewandte Seite der Gegenstände vorstellen zu wollen, diese mitzudenken und in die konkrete wissenschaftliche Arbeit einzubeziehen. Mit anderen Worten: Multiperspektivität nicht zu fordern, sondern zu erproben, so konsequent wie möglich. Wir sähen darin einen Gewinn für das in Abschn. 3.2 erläuterte Konzept soziologischer Wissenskulturen im Allgemeinen 
sowie die wissenskulturelle Reflexion einer Soziologie sozialer Probleme im Besonderen.

3. Angesprochen ist damit drittens das Moment der Reflexivität, das sich durch alle drei in diesem Beitrag skizzierten Konzeptionen hindurchzieht, das aber - wenn man so will - wissenspolitische Implikationen auch im Hinblick auf wissenschaftliches Denken und Handeln hat. Die Soziologie insgesamt, und damit auch Wissenssoziologie und Soziologie sozialer Probleme treffen in ihrem Untersuchungsfeld zunehmend auf eine soziologisch expertisierte Praxis, auf ihre eigene Geschichte und Positionierung. Das stellt sie auf Dauer vor die Herausforderung, sich selbst neu erfinden, also in Differenz zu diesem Vorgefundenen bringen zu müssen, um ,interessant ‘ zu bleiben. Die darin eingehegte Dynamik einer sich ,absetzenden' Wissensproduktion wird deutlich, wenn soziale Probleme in ihrem Gegenstandsfeld bereits von soziologischem Wissen konstituiert, erfasst, beschrieben werden. Das Verhältnis von wissenschaftlicher Disziplin und gesellschaftlichem Gegenstand wird in solchen Konstellationen grundlegend neu bestimmt. Reflexivität taucht hier ebenso sehr als strukturell angelegte Selbstbegegnung im Sinne von Ulrich Beck wie als Wissenszuwachs und Expertisierung im Sinne von Anthony Giddens auf. Damit stellt sich für Wissenssoziologie wie für die Soziologie sozialer Probleme die Grundfrage, was sie weiterhin ,wissenswert' macht - mit anderen Worten: wie sie also in der Lage ist, analytisch überraschende Neubeschreibungen des Sozialen zu generieren.

Funding Open Access funding enabled and organized by Projekt DEAL.

Open Access Dieser Artikel wird unter der Creative Commons Namensnennung 4.0 International Lizenz veröffentlicht, welche die Nutzung, Vervielfältigung, Bearbeitung, Verbreitung und Wiedergabe in jeglichem Medium und Format erlaubt, sofern Sie den/die ursprünglichen Autor(en) und die Quelle ordnungsgemäß nennen, einen Link zur Creative Commons Lizenz beifügen und angeben, ob Änderungen vorgenommen wurden.

Die in diesem Artikel enthaltenen Bilder und sonstiges Drittmaterial unterliegen ebenfalls der genannten Creative Commons Lizenz, sofern sich aus der Abbildungslegende nichts anderes ergibt. Sofern das betreffende Material nicht unter der genannten Creative Commons Lizenz steht und die betreffende Handlung nicht nach gesetzlichen Vorschriften erlaubt ist, ist für die oben aufgeführten Weiterverwendungen des Materials die Einwilligung des jeweiligen Rechteinhabers einzuholen.

Weitere Details zur Lizenz entnehmen Sie bitte der Lizenzinformation auf http://creativecommons.org/ licenses/by/4.0/deed.de.

\section{Literatur}

Abbott, Andrew. 2001. Chaos of disciplines. Chicago: University of Chicago Press.

Adorjan, Michael. 2019a. Constructionism Re-examined. Thematic Issue. The American Sociologist 50(2). Adorjan, Michael. 2019b. Social constructionism now more than ever: following the hermeneutic money trail in a post-truth world. The American Sociologist 50(2):160-174.

Akagawa, Manabu. 2015. Regulating pornocomic sales to juveniles in Japan: cycles and path-dependence of a social problem. Qualitative Sociology Review 11(2):62-73. http://www.qualitativesociologyreview. org/ENG/archive_eng.php. Zugegriffen: 25. September 2020.

Akagawa, Manabu. 2019. A natural history model of low birth rate issues in Japan since the 1990s. The American Sociologist 50(2):300-314. 
Albrecht, Günter. 2000. Konstruktion von Realität und Realität von Konstruktion. Soziale Probleme 12(1/2):116-145.

Beck, Ulrich. 1986. Risikogesellschaft. Auf dem Weg in eine andere Moderne. Frankfurt am Main: Suhrkamp.

Beck, Ulrich. 1988. Gegengifte. Die organisierte Unverantwortlichkeit. Frankfurt am Main: Suhrkamp.

Beck, Ulrich. 2004. Der kosmopolitische Blick oder: Krieg ist Frieden. Frankfurt am Main: Suhrkamp.

Beck, Ulrich. 2017. Die Metamorphose der Welt. Berlin: Suhrkamp.

Beck, Ulrich, und Edgar Grande. Hrsg. 2010. Varieties of Second Modernity: Extra-European and European Experiences and Perspectives. Special Issue. British Journal of Sociology 61(3).

Beck, Ulrich, und Christoph Lau. 2005. Second modernity as a research agenda: theoretical and empirical explorations in the "meta-change" of modern society. British Journal of Sociology 56(4):525-557.

Beck, Ulrich, Anthony Giddens, und Scott Lash. 1996. Reflexive Modernisierung. Eine Kontroverse. Frankfurt am Main: Suhrkamp.

Beck, Ulrich, Wolfgang Bonß, und Christoph Lau. 2001. Theorie reflexiver Modernisierung - Fragestellungen, Hypothesen, Forschungsprogramme. In Die Modernisierung der Moderne, Hrsg. Ulrich Beck, Wolfgang Bonß, 11-59. Frankfurt am Main: Suhrkamp.

Becker, Howard S. (Hrsg.). 1966. Social problems. A modern approach. New York: John Wiley.

Becker, Howard S. 2014a. Außenseiter, 2. Aufl., Wiesbaden: Springer VS.

Becker, Howard S. 2014b. What about Mozart? What about murder? Reasoning from cases. Chicago: University of Chicago Press.

Becker, Howard S. 2017. Evidence. Chicago: University of Chicago Press.

Bereswill, Mechthild, Anke Neuber, und Albert Scherr. 2020. Axel Groenemeyer und die Soziologie Sozialer Probleme. Ein Nachruf. Zeitschrift für Soziologie 49(4):273-275.

Berger, Peter L., und Thomas Luckmann. 1981. Die gesellschaftliche Konstruktion der Wirklichkeit. Eine Theorie der Wissenssoziologie. Frankfurt am Main: Fischer.

Best, Joel. 1993. But seriously folks. The limitations of the strict constructionist interpretation of social problems. In Reconsidering social constructionism. Debates in social problems theory, Hrsg. James A. Holstein, Gale Miller, 129-147. Hawthorne: Aldine de Gruyter.

Best, Joel. 2016. Social problems, 3. Aufl., New York: W.W. Norton \& Company.

Blumer, Herbert. 1971. Social problems as collective behavior. Social Problems 18(3):298-306.

Boltanski, Luc. 1999. Distant suffering. Morality, media and politics. Cambridge: Cambridge University Press.

Boltanski, Luc, und Laurent Thévenot. 2007. Über die Rechtfertigung. Eine Soziologie der kritischen Urteilskraft. Hamburg: Hamburger Edition.

Cohen, Stanley. 2002. Folk devils and moral panics. The creation of mods and rockers, 3. Aufl., London: Routledge.

Dello Buono, Ricardo A. 2015. Reimagining social problems: moving beyond social constructionism. Social Problems 62(3):331-342.

Dimbath, Oliver, und Reiner Keller. 2017. Was ist Wissenssoziologie? Zur Orientierung in einem unübersichtlichen Forschungsgebiet. Soziopolis 30(5). https://www.soziopolis.de/verstehen/was-tut-diewissenschaft/artikel/was-ist-wissenssoziologie/. Zugegriffen: 25.11.2020.

Dimbath, Oliver, und Michaela Pfadenhauer (Hrsg.). 2021. Gewissheit. Beiträge und Debatten zum 3. Sektionskongress der Wissenssoziologie. Weinheim: Beltz Juventa. im Druck.

Eisenstadt, Shmuel N. 2000. Multiple modernities. Daedalus 129(1):1-29.

Giddens, Anthony. 1984. Interpretative Soziologie. Frankfurt am Main: Suhrkamp.

Groenemeyer, Axel. 2001a. Soziale Probleme - Konstruktivistische Kontroversen und gesellschaftliche Herausforderungen. Soziale Probleme 12(1/2).

Groenemeyer, Axel. 2001b. Soziale Probleme - Konstruktivistische Kontroversen und gesellschaftliche Herausforderungen. Eine Einführung. Soziale Probleme 12(1/2):5-26.

Groenemeyer, Axel. 2007. Die Institutionalisierung von Problemdiskursen und die Relevanz der Soziologie sozialer Probleme. Soziale Probleme 18(1):5-25.

Groenemeyer, Axel. 2010. Doing Social Problems. Mikroanalysen der Konstruktion sozialer Probleme und sozialer Kontrolle in institutionellen Kontexten. Wiesbaden: VS.

Groenemeyer, Axel. 2012. Soziologie sozialer Probleme - Fragestellungen, Konzepte und theoretische Perspektiven. In Handbuch Soziale Probleme, 2. Aufl., Hrsg. Günter Albrecht, Axel Groenemyer, 17-116. Wiesbaden: Springer VS.

Groenemeyer, Axel, Christoph Hohage, und Melanie Ratzka. 2012. Die Politik sozialer Probleme. In Handbuch Soziale Probleme, Hrsg. Günter Albrecht, Axel Groenemyer, 117-194. Wiesbaden: Springer VS. 
Gusfield, Joseph R. 1981. The culture of public problems. Drinking driving and the symbolic order. Chicago: University of Chicago Press.

Gusfield, Joseph R. 1983. Symbolic crusade: status politics and the American temperance movement. Urbana-Champaign: University of Illinois Press.

Hacking, Ian. 1999. Was heißt „soziale Konstruktion“? Zur Konjunktur einer Kampfvokabel in den Wissenschaften. Frankfurt am Main: Fischer.

Hall, Stuart, Chas Critcher, Tony Jefferson, John Clarke, und Brian Roberts. 2013. Policing the crisis: mugging, the state, and law and order. London: Red Globe Press.

Haraway, Donna. 1988. Situated knowledges: the science question in feminism and the privilege of partial perspectives. Feminist Studies 14:575-599.

Harding, Sandra. 2003. The feminist standpoint theory reader. Intellectual and political controversies. London: Routledge.

Hitzler, Ronald. 1993. Verstehen: Alltagspraxis und wissenschaftliches Programm. In „Wirklichkeit“ im Deutungsprozeß: Verstehen und Methoden in den Kultur- und Sozialwissenschaften, Hrsg. Thomas Jung, Stephan Müller-Doohm, 223-240. Frankfurt am Main: Suhrkamp.

Hitzler, Ronald. 1999. Konsequenzen der Situationsdefinition. Auf dem Weg zu einer selbstreflexiven Wissenssoziologie. In Hermeneutische Wissenssoziologie. Standpunkte zur Theorie der Interpretation, Hrsg. Ronald Hitzler, Jo Reichertz, und Norbert Schröer, 289-308. Konstanz: UVK.

Hitzler, Ronald, Jo Reichertz, und Norbert Schröer (Hrsg.). 1999. Hermeneutische Wissenssoziologie. Standpunkte zur Theorie der Interpretation. Konstanz: UVK.

Hitzler, Ronald, Jo Reichertz, und Norbert Schröer (Hrsg.). 2020. Kritik der Hermeneutischen Wissenssoziologie. Weinheim: Beltz Juventa.

Keller, Reiner. 2011. Wissenssoziologische Diskursanalyse. Grundlegung eines Forschungsprogramms, 3. Aufl., Wiesbaden: Springer VS.

Keller, Reiner. 2017. Neuer Materialismus und Neuer Spiritualismus? Diskursforschung und die Herausforderung der Materialitäten. Österreichische Zeitschrift für Volkskunde Neue Serie 120(1/2):5-32.

Keller, Reiner. 2019a. Die Untersuchung von Dispositiven. Zur fokussierten Diskurs- und Dispositivethnografie in der Wissenssoziologischen Diskursanalyse. In Diskursive Konstruktionen. Kritik, Materialität und Subjektivierung in der wissenssoziologischen Diskursforschung, Hrsg. Saša Bosančić, Reiner Keller, 51-74. Wiesbaden: Springer VS.

Keller, Reiner. 2019b. New materialism? A view from sociology of knowledge. In Discussing new materialism. Methodological implications for the study of materialities, Hrsg. Ulrike T. Kissmann, Joost van Loon, 151-172. Wiesbaden: Springer VS.

Keller, Reiner, Hubert Knoblauch, und Jo Reichertz (Hrsg.). 2013. Kommunikativer Konstruktivismus. Theoretische und empirische Arbeiten zu einem neuen wissenssoziologischen Ansatz. Wiesbaden: Springer VS.

Knoblauch, Hubert. 2014. Wissenssoziologie, 3. Aufl., Konstanz: UVK/Utb.

Knoblauch, Hubert. 2016. Die kommunikative Konstruktion der Wirklichkeit. Wiesbaden: Springer VS.

Knoblauch, Hubert, und Michaela Pfadenhauer. 2019. Social constructivism as paradigm? The legacy of the social construction of reality. London: Routledge.

Knorr Cetina, Karin. 1989. Spielarten des Konstruktivismus. Soziale Welt 40(1/2):69-79.

Knorr Cetina, Karin. 2002. Wissenskulturen. Ein Vergleich naturwissenschaftlicher Wissensformen. Frankfurt am Main: Suhrkamp.

Kovach, Margaret. 2009. Indigenous methodologies: characteristics, conversations, and contexts. Toronto: University of Toronto Press.

Krohn, Wolfgang. 2007. Realexperimente - Die Modernisierung der ,offenen Gesellschaft' durch experimentelle Forschung. Erwägen-Wissen-Ethik (EWE) 18(3):343-356. Kritiken: 357-426. Replik des Autors: 427-442.

Lather, Patti, und Elizabeth St Pierre. 2013. Post-qualitative research. International Journal of Qualitative Studies in Education 26(6):629-633.

Loseke, Donileen R. 2015. Introduction to constructionist futures: new directions in social problems theory. Qualitative Sociology Review 11(2):6-14. http://www.qualitativesociologyreview.org/ENG/archive_ eng.php. Zugegriffen: 25. September 2020.

Luckmann, Thomas. 2010. „Ich habe mich nie als Konstruktivist betrachtet“. Gespräch mit Thomas Luckmann. In Soziologie in Frankfurt. Eine Zwischenbilanz, Hrsg. Felicia Herrschaft, Klaus Lichtblau, 345-368. Wiesbaden: VS.

Lukács, Georg. 1970. Geschichte und Klassenbewußtsein. Studien über marxistische Dialektik. Neuwied, Berlin: Luchterhand.

Mannheim, Karl. 1964. Wissenssoziologie. Auswahl aus dem Werk. Neuwied, Berlin: Luchterhand. 
Mead, George H. 1934. Democracy and universality in society. In Mind self and society from the standpoint of a social behaviorist, Hrsg. Charles W. Morris, 281-289. Chicago: University of Chicago Press.

Merton, Robert K. 1973. The sociology of science. Theoretical and empirical investigations. Chicago: University of Chicago Press.

Mignolo, Walter D., und Catherine E. Walsh. 2018. On decoloniality. Concepts, analytics, praxis. Durham, London: Duke University Press.

Mills, Charles Wright. 1940. Situated actions and the vocabularies of motives. American Sociological Review 5(6):904-913.

Mohanty, Chandra Talpade. 2003. Feminism without borders. Decolonizing theory, practicing solidarity. Durham, London: Duke University Press.

Nichols, Lawrence T. 2019. Editor's introduction: constructionism as interpretive tradition and as emergent deviance in sociology. The American Sociologist 50(2):155-159.

Poferl, Angelika. 1999. Gesellschaft im Selbstversuch. Der Kick am Gegenstand oder: Zu einer Perspektive ,experimenteller Soziologie‘. Soziale Welt 50(4):29-38.

Poferl, Angelika. 2010. Jenseits der Solidarität? Globale Probleme und die kosmopolitische Konstitution von Sozialität. In Große Armut, großer Reichtum. Zur Transnationalisierung sozialer Ungleichheit, Hrsg. Ulrich Beck, Angelika Poferl, 134-167. Berlin: Suhrkamp.

Poferl, Angelika. 2013. Problematisierungswissen und die Konstitution von Globalität. In Transnationale Vergesellschaftungen. Verhandlungen des 35. Kongresses der Deutschen Gesellschaft für Soziologie in Frankfurt am Main 2010, Teil 1, Hrsg. Hans-Georg Soeffner, 610-632. Frankfurt am Main, New York: Campus.

Poferl, Angelika. 2015. Gender und die Soziologie der Kosmopolitisierung. In Zeitgenössische Gesellschaftstheorien und Geschlechterforschung. Einladung zum Dialog, 2. Aufl., Hrsg. Heike Kahlert, Christine Weinbach, 127-151. Wiesbaden: Springer VS.

Poferl, Angelika. 2016. Die Kosmopolitisierung von Sozialität und Subjektivität. Zur Wahrnehmung globaler Probleme im Rahmen einer Kultur der Menschenrechte. In Subjekt - Handeln - Institution: Vergesellschaftung und Subjekt in der reflexiven Moderne, Hrsg. Fritz Böhle, Werner Schneider, 188-213. Weilerswist: Velbrück.

Poferl, Angelika. 2018. Cosmopolitan entitlements. Human rights and the constitution of human beings as human rights subjects. Transnational Social Review 8(1):79-92.

Poferl, Angelika. 2020. How to talk about difference and equality? Human dignity, gender, and the cosmopolitics of the social. In Multiple Gender Cultures, Sociology, and Plural Modernities, Hrsg. Heidemarie Winkel, Angelika Poferl. London: Routledge. im Druck.

Poferl, Angelika, und Reiner Keller. 2018. Form und Feld. Soziologische Wissenskulturen zwischen diskursiver Strukturierung und erkenntnisorientiertem Handeln. In Reihe Wissenskulturen Wissenskulturen der Soziologie, Bd. 1, Hrsg. Reiner Keller, Angelika Poferl, 18-39. Weinheim: Beltz Juventa.

Poferl, Angelika, und Michaela Pfadenhauer (Hrsg.). 2018. Wissensrelationen. Beiträge und Debatten zum 2. Sektionskongress der Wissenssoziologie. Weinheim: Beltz Juventa.

Poferl, Angelika, und Jo Reichertz (Hrsg.). 2015. Wege ins Feld: Methodologische Aspekte des Feldzugangs. Essen: Oldib.

Poferl, Angelika, und Norbert Schröer (Hrsg.). 2014. Wer oder was handelt? Zum Subjektverständnis der Hermeneutischen Wissenssoziologie. Wiesbaden: Springer VS.

Poferl, Angelika, und Verena Walter. 2013. „Deine Stimme gegen Armut“ - Zur kommunikativen Konstruktion eines globalen Problems. In Kommunikativer Konstruktivismus. Theoretische und empirische Arbeiten zu einem neuen wissenssoziologischen Ansatz, Hrsg. Reiner Keller, Hubert Knoblauch, und Jo Reichertz, 235-256. Wiesbaden: Springer VS.

Raab, Jürgen, und Reiner Keller (Hrsg.). 2016. Wissensforschung - Forschungswissen. Verhandlungen des ersten Sektionskongresses der Sektion Wissenssoziologie. Weinheim: Beltz Juventa.

Reichertz, Jo. 2010. Kommunikationsmacht. Wiesbaden: Springer VS.

Sarbin, Theodore R., und John I. Kitsuse (Hrsg.). 1994a. Constructing the social. London: SAGE.

Sarbin, Theodore R., und John I. Kitsuse. 1994b. A prologue to constructing the social. In Constructing the social, Hrsg. Theodore R. Sarbin, John I. Kitsuse, 1-18. London: SAGE.

Schetsche, Michael. 2000. Wissenssoziologie sozialer Probleme. Grundlegung einer relativistischen Problemtheorie. Opladen: Westdeutscher Verlag.

Schetsche, Michael. 2014. Empirische Analyse sozialer Probleme - Das wissenssoziologische Programm, 2. Aufl., Wiesbaden: Springer VS.

Schütz, Alfred. 1982. Das Problem der Relevanz. Frankfurt am Main: Suhrkamp.

Schütz, Alfred, und Thomas Luckmann. 1979. Strukturen der Lebenswelt. Bd. 1. Frankfurt am Main: Suhrkamp. 
Soeffner, Hans Georg. 1989. Auslegung des Alltags - Der Alltag der Auslegung. Zur wissenssoziologischen Konzeption einer sozialwissenschaftlichen Hermeneutik. Frankfurt am Main: Suhrkamp.

Soeffner, Hans-Georg. 2000. Gesellschaft ohne Baldachin. Über die Labilität von Ordnungskonstruktionen. Weilerswist: Velbrück.

de Sousa Santos, Boaventura. 2014. Epistemologies of the south. Justice against epistemicide. London: Paradigm Publishers, Boulder.

Spector, Malcolm. 2019. Constructing social problems forty years later. The American Sociologist 50(2):175-181.

Spector, Malcolm, und John I. Kitsuse. 1977. Constructing social problems. Menlo Park: Cummings Pub. Taylor, Charles. 1996. Quellen des Selbst. Die Entstehung der neuzeitlichen Identität. Frankfurt am Main: Suhrkamp.

Ulrich, Peter, und Reiner Keller. 2014. Comparing discourse between cultures. A discursive approach to movement knowledge. In Conceptualizing culture in social movement research, Hrsg. Britta Baumgarten, Priska Daphi, und Peter Ulrich, 113-139. Hampshire: Palgrave.

Winkel, Heidemarie, und Angelika Poferl (Hrsg.). 2020. Multiple gender cultures, sociology, and plural modernities. London: Routledge. im Druck. 